\title{
CIRCLE PACKING WITH GENERALIZED BRANCHING
}

\author{
JAMES ASHE, EDWARD CRANE AND KENNETH STEPHENSON
}

Dedicated to C. David Minda on the occasion of his retirement

Classical analytic function theory is at the heart of David Minda's research and of many of the results in this volume. It has been a pleasure in recent years to find that simple patterns of circles called circle packings could find themselves in such tight company with this classical theory. David himself contributed to this topic in [7] and on his retirement will surely have time to dive back into it.

Let us briefly review the circle packing story line. It began with Bill Thurston's observation that for every abstract triangulation $K$ of a topological sphere there exists an essentially unique configuration of circles with mutually disjoint interiors on the Riemann sphere $\mathbb{P}$ whose pattern of tangencies is encoded in $K$. That is, there's a circle packing $P$ for $K$ in $\mathbb{P}$. Based on this rigidity and his intuition, Thurston made a remarkable proposal at the 1985 Conference in Celebration of de Branges' Proof of the Bieberbach Conjecture: namely, that one could use such circle packings to approximate conformal mappings. The subsequent proof of his conjecture by Burt Rodin and Dennis Sullivan [8] established circle packing as a topic and opened its most widely known aspect, the approximation of classical analytic functions.

As this approximation theory developed, a second aspect that we will call discrete analytic function theory, began to emerge. For it became increasingly clear that classical phenomena were already at play within circle packing - mappings between circle packings not only approximated analytic functions, they also mimicked them. The literature shows an ever growing list of conformal notions being realized discretely and often with remarkable geometric fidelity: moving circle packing into the hyperbolic geometry of $\mathbb{D}$ led to infinite packings and the consequent classical type conditions the spherical, hyperbolic, and euclidean trichotomy - and from that came the discrete uniformization theorem, discrete Riemann surfaces and covering theory, random walks, and then notions of branch points and boundary conditions allowed for discrete versions of familiar classes of functions, polynomials, exponentials, and the Blaschke products, Ahlfors functions, and Weierstrass functions that play their roles in this paper.

Date: July 13, 2016. 
Part and parcel in these developments has been a third aspect, computation. Circle packings demand to be seen; that has led to packing algorithms, followed by experiments, then new - often surprising - observations, augmented theory, more computations, on and on. The work here was motivated by computational challenges, and the images behind our work are produced with the open software package CirclePack, $[10$.

Step after step in this story one can observe the remarkable faithfulness of the discrete theory to its continuous precedents so that today one can claim a fairly comprehensive discrete world parallel to the classical world of analytic functions (and invariably converging to it in the limit as the combinatorics are refined). Yet this discrete world can never be fully comprehensive, one always faces "discretization issues". This paper is a preliminary description of new machinery for addressing the principal remaining gap in the foundation of discrete function theory, the existence and uniqueness of discrete meromorphic functions. The sphere is a difficult setting for circle packing. On the practical side, there is no known algorithm for computing circle packings in situ, restricting the experimental approach; essentially all circle packings on $\mathbb{P}$ have been obtained via the stereographic projection of hyperbolic or euclidean packings. More crucially, the compactness of the sphere brings conformal rigidity, with topologically mandated branching and no boundary to provide maneuvering room.

Branching difficulties are the discretization issue we address here. We introduce generalized branching, which began with the thesis of the first author, 1]. We believe general branching will provide the flexibility necessary to construct the full spectrum of discrete branched mappings while keeping two main objectives at the fore: (1) discrete analytic functions should display qualitative behaviors parallel to their classical counterparts, and (2) discrete analytic functions should converge under refinement to their classical counterparts.

\section{Classical Models}

We use three types of classical functions to motivate this work: finite Blaschke products on the unit disc, Ahlfors functions on annuli, and Weierstrass functions on tori. We review these in preparation for their discrete versions.

Blaschke Products: A classical finite Blaschke product $\mathbf{B}: \mathbb{D} \rightarrow \mathbb{D}$ is a proper analytic self map of the unit disc $\mathbb{D}$. In particular, $\mathbf{B}$ has finite valence $N \geq 1$, it maps the unit circle $N$ times around itself, and it has $N-1$ branch points in $\mathbb{D}$, counting multiplicities - that is $\mathbf{B}^{\prime}$ has $N-1$ zeros in $\mathbb{D}$. The function $\mathbf{B}$ is known as an $N$-fold Blaschke product. Topologically 
speaking, B maps $\mathbb{D}$ onto an $N$-sheeted complete branched covering of $\mathbb{D}$. The images of the branch points under $\mathbf{B}$ are known as branch values.

As a concrete example, let us distinguish two points $p_{1} \neq p_{2}$ in $\mathbb{D}$. It is well known that there exists a 3 -fold Blaschke product $\mathbf{B}$ with $p_{1}, p_{2}$ as simple branch points. It is convenient to assume a standard normalization, so by post-composing with a conformal automorphism (Möbius transformation) of $\mathbb{D}$ we may arrange further that $\mathbf{B}(0)=0$ and $\mathbf{B}(i)=i$. This is the function we will have in mind for discretization later.

Ahlfors Functions: Our next model is defined on a proper annulus $\Omega$. By standard conformal mapping arguments, we may take $\Omega$ to be a standard annulus, $\Omega=\{z: r<|z|<1 / r\}$, with $0<r<\infty$. Designating a point $z_{0} \in \Omega$, one may consider the extremal problem: maximize $\left|\mathbf{F}^{\prime}\left(z_{0}\right)\right|$ over all analytic functions $\mathbf{F}: \Omega \rightarrow \mathbb{D}$. The solution $\mathbf{A}(z)$ is known to exist, is unique up to multiplication by a unimodular constant, and is referred to as an Ahlfors function for $\Omega$. Ahlfors functions are also characterized, however, by their mapping properties. They are the proper analytic mappings $\mathbf{A}: \Omega \rightarrow \mathbb{D}$ which extend continuously to $\partial \Omega$ and map each component of $\partial \Omega$ 1-to- 1 onto the unit circle. Any such map will be a branched double covering of $\mathbb{D}$ with two simple branch points, $p_{1}, p_{2} \in \Omega$. It is fundamental to function theory on $\Omega$ and is analogous to the 1 -fold Blaschke products on $\mathbb{D}$, i.e., Möbius transformations. The Ahlfors function for $\Omega$ is determined uniquely by $r$ (up to pre- and post-composition by conformal automorphisms).

To have a concrete example in mind for discretization, let us suppose that $z_{0}$ is on the midline of $\Omega$, say $z_{0}=1$. From elementary symmetry considerations we deduce that $\mathbf{A}(1)=\mathbf{A}(-1)=0$ and that the branch points in $\Omega$ lie at $p_{1}=i$ and $p_{2}=-i$. A normalization in the range, $\mathbb{D}$, will put the branch values on the imaginary axis, symmetric with respect to the origin.

Weierstrass Functions: Our final model is the classical Weierstrass function W. This is a meromorphic function mapping a conformal torus $T$ to a branched double covering of the sphere. A fundamental domain for $T$ is the parallelogram in $\mathbb{C}$ with corners $0,1, \tau, 1+\tau$, where $\tau$, a complex number in the upper half plane, is the so-called modulus of $T$. The function $\mathbf{W}$ has four simple branch points at $0,1 / 2, \tau / 2$, and $(1+\tau) / 2$ and is determined uniquely by $\tau$ (up to pre- and post-composition by conformal automorphisms).

Note that while all three classes of functions are characterized by their topological mapping properties, only with the Blaschke products do we get any choice in the branch points - for Ahlfors and Weierstrass functions, branch point locations are (up to normalization) forced on us by the conformal geometry of the domain. 


\section{Discrete Versions}

We will now describe and illustrate discrete versions of these classical functions. We assume a basic familiarity with circle packing, as presented in 11 for example. However, a brief overview might help, and with the images here should aid the intuition, even for those not familiar with details.

A discrete analytic function is a map between circle packings. The domain, rather than being a Riemann surface, will now be a triangulated topological surface with combinatorics encoded as a simplicial 2-complex $K$ : thus, we will be selecting $K$ to be a combinatorial disc, a combinatorial annulus, or a combinatorial torus, as appropriate. A circle packing for $K$ is a configuration $P$ of circles, $P=\left\{c_{v}\right\}$ with a circle $c_{v}$ associated with each vertex $v$ of $K$. The circle packing may live in the euclidean plane, $\mathbb{C}$, in the hyperbolic plane, represented as the unit disc $\mathbb{D}$, or on the Riemann sphere, $\mathbb{P}$. The only requirements are that whenever $\langle v, w\rangle$ is an edge of $K$, then circles $c_{v}, c_{w}$ must be (externally) tangent, and when $\langle v, u, w\rangle$, is an oriented face of $K$, then the circles $c_{v}, c_{u}, c_{w}$ must form an oriented triple of mutually tangent circles. The carrier of $P$, denoted $\operatorname{carr}(P)$, is the polyhedral surface formed by connecting the centers of tangent circles with geodesic segments; that is, $\operatorname{carr}(P)$ is an immersion of the abstract triangulation $K$ as a concrete triangulated surface.

At the foundation of the theory is the fact that each complex $K$ has a canonical maximal packing $P_{K}=\left\{C_{v}: v \in K\right\}$. This is a univalent circle packing, meaning the circles have mutually disjoint interiors, which fills $\mathbb{D}$, a conformal annulus, or a conformal torus, as the case may be. The packing $P_{K}$ serves as the domain for discrete analytic functions associated with $K$. The image will be a second circle packing $P$ for $K$ which lies in $\mathbb{D}$ for discrete Blaschke products and discrete Ahlfors functions, or on the sphere $\mathbb{P}$ for discrete Weierstrass functions. The discrete analytic function, then, will be the map f : $P_{K} \rightarrow P$ which identifies corresponding circles. (One may also treat $\mathbf{f}$ as a topological mapping $\mathbf{f}: \operatorname{carr}\left(P_{K}\right) \rightarrow \operatorname{carr}(P)$ by mapping circle centers to circle centers and extending via barycentric coordinates to edges and faces.)

We are now ready for the discrete constructions. Central to our work is the issue of branching, as we will see in this first discrete example.

2.1. Discrete Blaschke Product. In a sense, discrete function theory began with the introduction of discrete Blaschke products; see [6] and [11, $\S 13.3]$. The construction here will serve to remind the reader of basic notation and terminology while providing an example directly pertinent to our work. 
A discrete finite Blaschke product $\mathbf{b}$ is illustrated in Figure 1, with the domain circle packing $P_{K}$ on the left and the image circle packing $P$ on the right, both in $\mathbb{D}$. There is nothing special in the underlying complex $K$, a combinatorial disc - it is just a generic triangulation of a topological disc, though there are minor combinatorial side conditions to avoid pathologies.

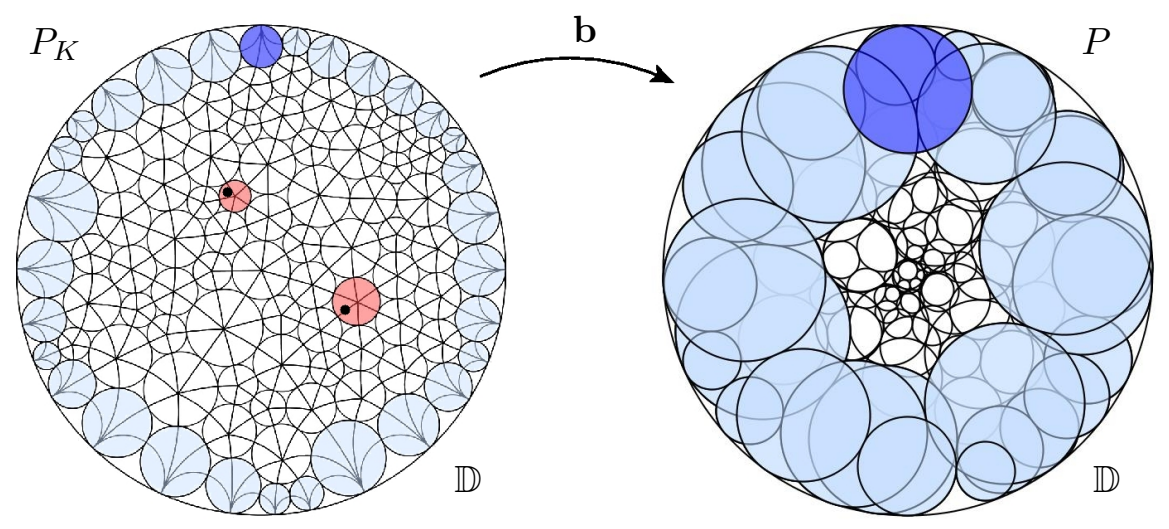

FiguRE 1. A 3-fold discrete Blaschke product $\mathbf{b}$, domain and range.

Begin with the domain packing for $\mathbf{b}$ on the left, the maximal packing $P_{K}=\left\{C_{v}: v \in K\right\}$. The boundary circles are horocyles (euclidean circles internally tangent to $\partial \mathbb{D}$ ). A designated interior vertex $\alpha$ has its circle $C_{\alpha}$ centered at the origin and a designated boundary vertex $\gamma$ has its circle $C_{\gamma}$ centered at $z=i$; the latter appears here as dark blue. The classical Blaschke product $\mathbf{B}$ discussed earlier involved branch points $p_{1}, p_{2}$; we assume these are the two black dots in the domain. To mimic this, we have identified interior circles $C_{v_{1}}, C_{v_{2}}$, red circles, whose centers are nearest to $p_{1}, p_{2}$, respectively.

Note that the unit disc is treated as the Poincaré model of the hyperbolic plane, so circle centers and radii are hyperbolic and the carrier faces are hyperbolic triangles. The boundary circles, as horocyles, are of infinite hyperbolic radius and have hyperbolic (ideal) centers at their points of tangency with the unit circle. The set of hyperbolic radii is denoted by $R_{K}=\left\{R_{K}(v)\right\}$. The existence of $P_{K}$ follows from the fundamental KoebeAndreev-Thurston Theorem, [11, Chp 6], as does its essential uniqueness up to conformal automorphisms of $\mathbb{D}$. In practice, however, it is computed based on angle sum conditions. The angle sum $\theta_{R_{K}}(v)$ at a vertex $v$ is the sum of angles at $v$ in all the faces to which it belongs and is easily computed from the radii $R_{K}$ using basic hyperbolic trigonometry. Clearly, one must have $\theta_{R_{K}}(v)=2 \pi$ for very interior $v$. This, along with the condition that $R_{K}(w)=\infty$ for boundary vertices $w$, is enough to solve for $R_{K}$. 
Let us now move to the more visually challenging range packing in Figure 1, denoted $P=\left\{c_{v}: v \in K\right\}$. This, too, is a hyperbolic circle packing for $K$, though it is clearly not univalent. We have arranged that the circle $c_{\alpha}$ is centered at the origin and that the circle $c_{\gamma}$ is a horocycle centered at $z=i$, just as in $P_{K}$. The boundary circles are again horocycles, and if one starts at $c_{\gamma}$ and follows the counterclockwise chain of successively tangent horocycles, one finds that they wraps three times about the unit circle. This mimics the behavior of our 3-fold classical Blaschke product $\mathbf{B}$.

The image of $P$ is a bit too fussy to show its carrier, but it is in fact a 3 -sheeted branched surface. Hidden among the interior circles of $P$ are the two associated with vertices $v_{1}, v_{2}$, the branch vertices. These circles, red in both domain and range, are difficult to pick out, but since branching is the central topic of the paper, we have blown up the local images at $v_{1}$ in Figure 2. We now describe what you are seeing.
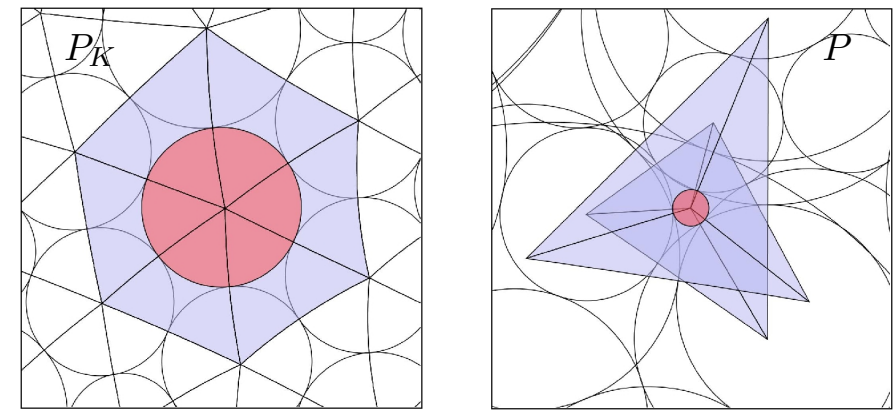

FiguRE 2. Isolated flowers for the branch vertex $v_{1}$ in the domain $P_{K}$ and range $P$ of the discrete Blaschke product $\mathbf{b}$.

This branching will be termed traditional; conceptually and computationally very simple, this method has, until now, provided all the branching for discrete function theory, [11, §11.3]. The flower for vertex $v$, the central circle (red) and its neighboring circles (its petals), are shown for $P_{K}$ on the left and for $P$ on the right. Whereas the six petals wrap once about $C_{v_{1}}$ in the domain, a careful check will show that they wrap twice around $c_{v_{1}}$ in the range. If $R$ denotes the set of hyperbolic radii for $P$, we may compute the angle sum $\theta_{R}\left(v_{1}\right)$ at $c_{v_{1}}$. Expressed in terms of angle sums, the branching is reflected in the fact that $\theta_{R_{K}}\left(v_{1}\right)=2 \pi$ in the domain, while $\theta_{R}\left(v_{1}\right)=4 \pi$ in the range. Mapping the faces about $C_{v_{1}}$ onto the corresponding faces about $c_{v_{1}}$ realizes a 2 -fold branched cover in a neighborhood of the center of $c_{v_{1}}$ - meaning a branched covering surface in the standard topological sense. Similar behavior could be observed locally at the other branch vertex, $v_{2}$, while at all other interior vertices the map between faces is locally univalent.

In summary, the circle packing map $\mathbf{b}: P_{K} \rightarrow P$ is called a discrete finite Blaschke product because it displays the salient mapping features of the 
classical Blaschke product $\mathbf{B}$ : namely, $\mathbf{b}$ is a self-map of $\mathbb{D}$, a 3-fold branched covering, it maps the unit circle 3 times about itself, and it harbors two interior branch points. We have even imposed the same normalization, $\mathbf{b}(0)=0$ and $\mathbf{b}(i)=i$. Additional features of such discrete analytic functions are developed in the relevant literature: Note in Figure 2 how much the circles for a branch vertex shrink under $\mathbf{b}$; this ratio of radii mimics the vanishing of the derivative at a branch point. Note in Figure 1 how $\mathbf{b}$ draws the interior circles together; this is the discrete hyperbolic contraction principle. Note that the circles for $\alpha$ are centered at the origin in both $P_{K}$ and $P$, but the latter is much smaller: this reflects the discrete Schwarz Lemma. On the other hand, the horocycle associated with $\gamma$ (blue) is much larger in $P$ than in $P_{K}$, reflecting the behavior of angular derivatives at the boundary. Discrete analytic function theory is rife with such parallel phenomena for a wide variety of situations, including the Ahlfors and Weierstrass examples to come.

2.2. Discrete Ahlfors Function. We build a clean example that mimics the classical Ahlfors function A described earlier. Our complex $K$ triangulates a topological annulus. Its maximal packing $P_{K}$ is represented in Figure 3 .

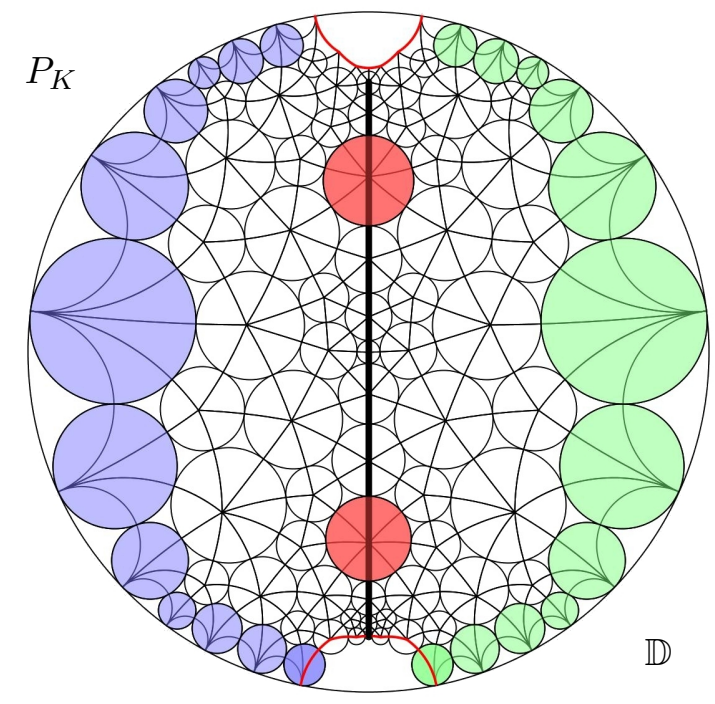

FIgURE 3. Maximal packing $P_{K}$ for a combinatorial annulus $K$, represented in a fundamental domain in $\mathbb{D}$.

A bit of explanation may help here: The maximal packing actually lives on a conformal annulus $\mathcal{A}$, with circles measured in its intrinsic hyperbolic metric. However, as $\mathbb{D}$ is the universal cover of $\mathcal{A}$, we can lift the packing to lie in a fundamental domain within $\mathbb{D}$ - that is what we see in Figure 3 . 
The boundary edges in red represent the lifts of a cross-cut of $\mathcal{A}$ and are identified by the hyperbolic Möbius transformation $\gamma$ of $\mathbb{D}$ which generates the covering group for $\mathcal{A}$. Applying $\gamma$ to the circles of Figure 3 , one would get new circles which blend seamlessly along the cross-cut.

We have chosen $K$ with foresight, as it displays two particularly helpful symmetries. The line in the center of Figure 3 marks the combinatorial midline of the annulus: $K$ is symmetric under reflection in this. Moreover, there is an order two translational symmetry along this midline; the automorphism $\sqrt{\gamma}$ will (modulo $\gamma$ ) carry $P_{K}$ to itself. Topology demands, as with the classical Ahlfors function $\mathbf{A}(z)$, that we have two simple branch points. Choose the midline vertices $v_{1}$ and $v_{2}$, their circles are red in Figure 3 , these two are fixed by the reflective symmetry and interchanged by the translational symmetry. Prescribing traditional branching at $v_{1}, v_{2}$ results in the branched circle packing, $P$, of Figure 4. The mapping a : $P_{K} \rightarrow P$ is thus a discrete analytic function from $\mathcal{A}$ to $\mathbb{D}$.

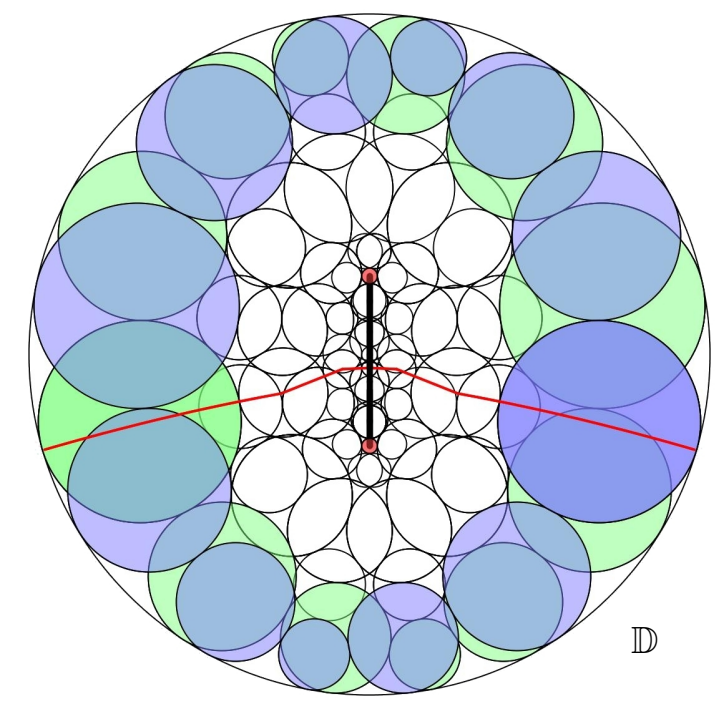

Figure 4. The branched packing $P$ for combinatorial annulus $K$.

Due to its mapping properties, we refer to a as a discrete Ahlfors function. In particular: The boundary circles of $P_{K}$ are horocycles; in Figure 3 , those on one boundary component are blue, those of the other, green. We would expect the boundary circles of $P$ to be horocycles as well, meaning that a maps each boundary component to the unit circle. With a careful look in Figure 4, one can disentangle the closed chain of blue horocycles reaching once around the unit circle and the second closed chain of green horocycles doing the same. The branch circles, $C_{v_{1}}, C_{v_{2}}$ in $P_{K}$, and their images, $c_{v_{1}}, c_{v_{2}}$ in $P$, are red. We have normalized by applying an automorphism to $\mathbb{D}$ that 
centers $c_{v_{1}}$ and $c_{v_{2}}$ on the imaginary axis and symmetric with respect to the origin. Thus, $P$ represents in a discrete way a double covering of $\mathbb{D}$ branched over two points. These are all hallmarks of the image of an Ahlfors function and mimic the classical function $\mathbf{A}$. For reference, in $P$ we have drawn in red the edges of $P$ corresponding to red cross-cut in $P_{K}$.

The computation of $P$ deserves special attention. Standard Perron methods allow one to compute a hyperbolic packing label $R$ for $K$ so that $R(w)=\infty$ for each boundary vertex $w \in K$ and angle sums $\theta_{R_{K}}\left(v_{j}\right)=4 \pi$ for $v_{1}, v_{2}$. There is nothing special in computing $R$. There is a second step, however: with $R$ in hand, one then lays out the circles in sequence and normalizes to get the packing $P$ of Figure 4. But why does this second step work so nicely? In circle packing, the laying out of circles is akin to analytic continuation of an analytic function element, and since $K$ is an annulus, its fundamental group is generated by some simple, closed, nonnullhomotopic loop $\Gamma$. Analytic continuation along $\Gamma$ would generically lead to a non-trivial holonomy: that is, given a function element $\mathfrak{f}$ defined at a point of $\Gamma$, one would anticipate a non-trivial automorphism $m$ of $\mathbb{D}$ so that analytic continuation of $\mathfrak{f}$ about $\Gamma$ would lead to a new element $m(\mathfrak{f}), m(\mathfrak{f}) \neq \mathfrak{f}$. In discrete terms, after laying out the circle $c_{v}$ for some vertex $v$ of $\Gamma$, and then laying out successively tangent circles for the vertices along $\Gamma$, one would not expect that upon returning to $v$ one would lay out the same circle $c_{v}$. Generically, there is a non-trivial automorphism $m$ so that upon returning to $v$ one lays out $m\left(c_{v}\right) \neq c_{v}$. As it happens here, things work out because of the symmetries built into $K$ - the holonomy $m$ is trivial, so the layout process results in a coherent branched circle packing $P$. The holonomy issue is key to later considerations.

2.3. Discrete Weierstrass Function. For this example our complex $K$ triangulates a topological torus. Its maximal packing $P_{K}$ is shown in Figure 5. Here again the maximal packing actually lives in a conformal torus $\mathcal{T}$ with its intrinsic euclidean metric. As $\mathbb{C}$ is the universal cover of $\mathcal{T}$, we may lift the packing to $\mathbb{C}$, and this lift is what we see on the left in Figure 5. This packs a fundamental domain, delineated by the color-coded edges, which represent the side-pairings.

We again have chosen $K$ with important symmetries. The four colored circles, symmetrically placed, are our chosen branch circles. The image packing $P$ on the sphere is shown on the right in Figure 5 two branched circles are visible on the front, the other two are (due to a normalization) antipodal to these. The result is a discrete analytic function $\mathbf{w}: P_{K} \rightarrow P$ which maps $\mathcal{T}$ to $\mathbb{P}$, that is, a discrete meromorphic function. Reprising its mapping properties, we are justified in calling $\mathbf{w}$ a discrete Weierstrass function. 


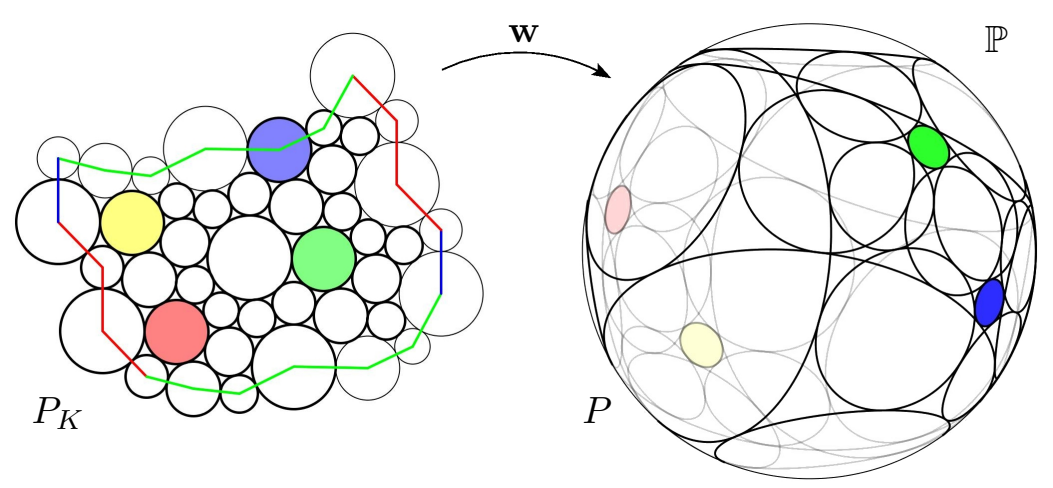

FiguRE 5. $P_{K}$ packs the fundamental domain for a combinatorial torus $K$ and shows four designated branch vertices and color-coded side pairings. $P$ is the branched image packing for $K$ on $\mathbb{P}$.

There is not yet a practical circle packing algorithm in spherical geometry, so the computation of $P$ takes a circuitous route. We puncture $K$ at one of the intended branch vertices, say $v_{4}$, and consider $K^{\prime}=K \backslash\left\{v_{4}\right\}$. This has a single boundary component, and the usual Perron arguments yield a hyperbolic packing label $R^{\prime}$ so that $R^{\prime}(w)=\infty$ for every boundary vertex $w$ and so that $\theta_{R^{\prime}}\left(v_{j}\right)=4 \pi, j=1,2,3$. Since $K^{\prime}$ has genus 1 , its fundamental group is again an obstruction to the layout process and a risk for nontrivial holonomy. However, symmetry saves us once more, and we obtain a coherent branched packing $P^{\prime}$ in $\mathbb{D}$ for $K^{\prime}$. Note, in particular, that the boundary circles of $P^{\prime}$ are horocycles, and topological counting arguments show that the chain of boundary horocycles must wrap twice around the unit circle. Stereographically projected to $\mathbb{P}, P^{\prime}$ lies in one hemisphere. The other hemisphere, treated as the inside of a circle, is tangent to the circles for all the former neighbors of $v_{4}$, so we simply declare this to be the circle for $v_{4}$. The neighbors wrap twice around, so this is the fourth branch point and, after a normalization, we arrive at $P$.

Note: Our methods clearly yield a coherent branched packing $P$ in this case, and have done the same in literally scores of similarly structured complexes. The key seems to lie with the symmetries in $K$ and in the branch set. We leave this as a Conjecture: If $K$ is a combinatorial torus with two commuting translational symmetries of order two and $\omega=\left\{v_{1}, v_{2}, v_{3}, v_{4}\right\}$ is an orbit of vertices under these symmetries, then traditional branching at the points of $\omega$ leads to trivial holonomy.

The good news is that we have successfully created discrete analogues for our three classical models: Blaschke products, Ahlfors functions, and Weierstrass functions. Let us now look into the bad news. 


\section{The Discretization Issue}

Whenever a continuous theory is discretized, whether in geometry, topology, differential equations, or $p$-adic analysis, problems will crop up. Replacing a continuous surface by a triangulated one, for example, leads to combinatorial restrictions. Thus a branch vertex must be interior and have at least 5 neighbor. We expect this. However, we are after a starker discretization effect. There are only finitely many possible locations for discrete branching. Our discrete Blaschke product could not branch precisely at the points $p_{1}, p_{2}$ prescribed for its classical model $\mathbf{B}$, and we instead chose to branch using the nearby circles $C_{v_{1}}$ and $C_{v_{2}}$. This effect is admittedly minor - the qualitative behavior of the discrete function is little affected by the misplaced branching. For the Ahlfors and Weierstrass cases, however, this problem is existential - discrete versions may fail to exist. We will illustrate the problem in the Ahlfors cases - and return to fix it in 86 .

Nearly any break in the combinatorial symmetries of the complex $K$ behind Figure 3 will cause the subsequent Ahlfors construction to fail. Most such failures will be difficult to fix, so we choose carefully: we make two small changes via edge flips so that we preserve the reflective symmetry but break the translational symmetry. The new complex will be denoted $K^{\prime}$. Repeating the Ahlfors construction from $\$ 2.2$ with $K^{\prime}$ and using the same $v_{1}, v_{2}$ as branch vertices gives the result of Figure 6 .

There is no difficulty in computing the branched packing label $R^{\prime}$ for $K^{\prime}$, however, the layout process does not give a coherent circle packing. The problem might be difficult to see in Figure 6, but look to the red edge paths, which correspond to layouts of the cross-cut: they are no longer coincident, as they were in Figure 4. One is a shifted copy of the other, reflecting a nontrivial holonomy associated with the generator of the fundamental group for $K^{\prime}$. More precisely, there is a non-trivial hyperbolic Möbius transformation $m$ of $\mathbb{D}$, which maps one of these red cross-cut curves onto the other. One would have to follow things very closely in the image to confirm the problem, but we illustrate with the two gray circles, which are supposed to be tangent to one another.

As it happens, no matter what pair of vertices of $K^{\prime}$ are chosen as branch points, the Ahlfors construction will fail - there will be no coherent image packing. It has been a long road to get to this point, but this is where our work begins: Our goal is to introduce generalized branching with the flexibility to make the discrete theory whole. We will illustrate it in action in $\$ 6$ by creating an Ahlfors function for this modified complex $K^{\prime}$. 


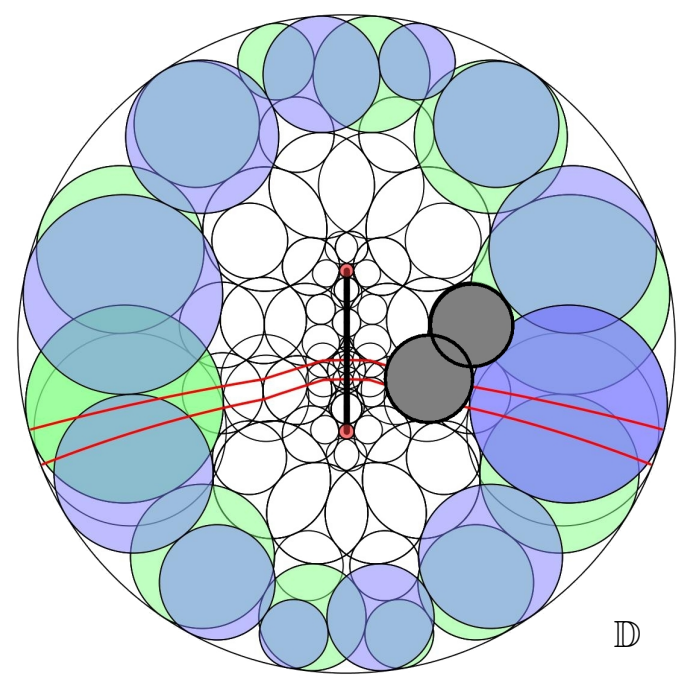

Figure 6. A failed attempt at an Ahlfors function using traditional branching. The non-trivial holonomy shows up in misalignment of the cross-cuts, and the failure of the gray circles to be tangent to one another.

\section{Generalized Branching}

Branching is perhaps most familiar in the analytic setting. Let $\mathbf{f}: G \rightarrow \mathbb{C}$ be a non-constant analytic function on an open domain $G \subset \mathbb{C}$. Suppose $z \in$ $G$ and $w=\mathbf{f}(z)$. For $\delta>0$, consider the disc $D=D(w, \delta)=\{\zeta:|\zeta-w|<\delta\}$ and the component of the preimage $U=\mathbf{f}^{-1}(D(w, \delta))$ containing $z$. For $\delta$ sufficiently small, $U$ will be a topological disc in $G$ and the restriction of $\mathbf{f}$ to the punctured disc $U^{\prime}=U \backslash\{z\}$ will be a locally 1-to-1 proper mapping onto the punctured disc $D^{\prime}=D \backslash\{w\}$. In particular, one can prove the existence of some $N \geq 1$ so that every point of $D^{\prime}$ has $N$ preimages in $U^{\prime}$. In this analytic case, if $N>1$, then $\mathbf{f}^{k}(z)=0$ for $k=1,2, \cdots, N-1$ and we say that $z$ is a branch point of order $N-1$ for $\mathbf{f}$. We refer to $w$ as its branch value.

This is, in fact, a topological phenomenon having little to do with analyticity: by Stoïlow's Theorem, [12, the same local behavior occurs whenever the map $\mathbf{f}$ is an open, continuous, light-interior mapping. In particular, this applies to our maps between the carriers of circle packings. One sees it on display for the traditional branch point illustrated in Figure 2 .

We can set the stage for generalized branching by simply enlarging the singleton set $\{z\}$ for a branch point to a compact topological disc $H$. If $H$ is small enough, then the mapping behavior in the neighborhood of $H$ is unchanged: that is, $\mathbf{f}$ will be a locally 1-to-1 proper map of the annulus 
$U^{\prime}=U \backslash H$ onto the annulus $D^{\prime}=D \backslash f(H)$ of valence $N$. When $N>1$ we will say that $\mathbf{f}$ has generalized branching of order $N-1$ in $H$. The point is that the branching is reflected in the mapping behavior between the annuli $U^{\prime}$ and $D^{\prime}$, even if the precise location of that branching is hidden within the hole $H$.

Let us apply this notion to the classical Blaschke product $\mathbf{B}$ discussed earlier. About each of its branch points $p_{j}, j=1,2$, we can choose a small compact topological disc $H_{j}$ and open neighborhood $U_{j}$ of $H_{j}$ so that $\mathbf{B}$ has generalized branching of order 1 in $H_{j}$. Making $H_{1}, H_{2}$ smaller, if necessary, we may assume $U_{1}$ and $U_{2}$ have disjoint closures. This leaves a triply connected open set $\Omega=\mathbb{D} \backslash\left\{\bar{U}_{1} \cup \bar{U}_{2}\right\}$. The restriction of $\mathbf{B}$ to $\Omega$ is a 3 -valent map onto $\mathbb{D}$ which maps $\partial U_{j}$ to a some curve about the branch value $\mathbf{B}\left(p_{j}\right), j=1,2$. If we were to perturb $p_{j}$ to a new point $\tilde{p}_{j}$ within $H_{j}, j=1,2$, then the associated finite Blaschke product $\widetilde{\mathbf{B}}$ would be qualitatively indistinguishable from $\mathbf{B}$ on $\Omega$ : that is, it is very difficult to discern where the branch points actually lie in $H_{1}, H_{2}$. This is the type of flexibility we need for discrete finite Blaschke products and motivates our strategy.

Given $K$ and its max packing $P_{K}$, we choose interior vertices $v_{1}, v_{2}$ so their circle centers are near $p_{1}, p_{2}$. Choose small combinatorial neighborhoods $H_{1}, H_{2}$ of $v_{1}, v_{2}$ and define $L=K \backslash\left\{H_{1} \cup H_{2}\right\}$, analogous to the open set $\Omega$ earlier. Requiring simple branching at $v_{1}$ and $v_{2}$ leads to the discrete Blaschke product $\mathbf{b}$ we discussed earlier. However, we have developed machinery, discussed in the next section, that allows us to modify the combinatorics and packing parameters inside $H_{1}$ and $H_{2}$. Patching these new combinatorics into $L$ gives a new combinatorial disc $\widetilde{K}$ on which we define a new discrete Blaschke product $\tilde{\mathbf{b}}$. The parameters involved allow us to perturb the apparent branch locations. In other words, just as with $\mathbf{B}$ and $\widetilde{\mathbf{B}}$ defined on $\Omega$, both $\mathbf{b}$ and $\tilde{\mathbf{b}}$ are defined on $L \subset K \cap \widetilde{K}$ and are qualitatively indistinguishable there.

Our global intention is to make adjustments in the small locales $H_{1}$ and $H_{2}$ so that $\tilde{\mathbf{b}}$ behaves like a discrete Blaschke product having branch points precisely at $p_{1}, p_{2}$. Mimicking this individual Blaschke product $\mathbf{B}$ may seem to be a lot of effort for little gain. However, if one thinks more broadly of the family of Blaschke products parameterized continuously by $p_{1}$ and $p_{2}$, the goal of continuously parameterized discrete versions makes more sense. It also makes more sense when the very existence of the discrete versions depends on this added flexibility, as with our broken Ahlfors example. Let us now describe the mechanics.

\section{LOCAL MECHANiCS}

We describe discrete generalized branching which takes two forms, termed singular and shifted branching. Each involves identifying a black hole $H$, a 
small combinatorial locale to support the branching, and its event horizon $\Gamma=\partial H$, the chain of surrounding edges. Outside of the event horizon, our circle packing mappings are defined in the usual way, so that in an annulus about the black hole one may observe the typical topological behavior described earlier. Adjustments hidden inside the black hole, however, allow our mapping to simulate simple branching at various points.

5.1. Background. We have recalled some circle packing mechanics, but as our work involves new features, we review the basics.

A complex $K$ is assumed to be given. The fundamental building blocks of $K$ are its triangles and flowers. The triangles are the faces $\langle v, u, w\rangle$. The flowers are sets $\left\{v ; v_{1}, v_{2}, \cdots, v_{n+1}\right\}$ where $v$ is a vertex and $v_{1}, \cdots, v_{n+1}$ is the counterclockwise list of neighbors in $K$. These neighbors, the petals, define the fan of faces containing $v$. Here $n$ is the number of faces, so when $v$ is interior, then $v_{n+1}=v_{1}$.

In talking about a circle packing $P$ for $K$, the radii and centers are, of course, the ultimate target. However, proofs of existence and uniqueness (and computations) depend on the standard Perron methods first deployed in 2. Given $K$, the fundamental data lies in three lists: the label $R=$ $\{R(v): v \in K\}$, edge overlaps $\Phi=\{\Phi(e): e=\langle u, v\rangle\}$, and target angle sums $A=\{A(v): v \in K\}$. Each will require some extension.

- Labels: The labels $R(v)$ are putative radii (they become actual radii only when a concrete packing is realized).

- Overlap Angles: For an edge $e=\langle v, w\rangle$ of $K$, the overlap $\Phi(e)$ represents the desired (external) angle between the circles $c_{v}, c_{w}$ in $P$. Interest is often in "tangency" packings; in this case, $\Phi$ is identically zero and hence does not appear explicitly. However, from Thurston's first introduction of circle packing, non-tangency packings were included and we need them here.

- Target Angle Sums: Given $R$ and $\Phi$, one can readily compute for any triangle $\langle u, v, w\rangle$ the angle which would be realized at $v$ if a triple of circles with the given labels (as radii) and edge overlaps were to be laid out. The angle sum $\theta_{R, \Phi}(v)$ is the sum of such angles for all faces containing $v$. The target angle sum, $A(v)$ is the intended value for $\theta_{R, \Phi}(v)$. It is typically prescribed only when $v$ is interior, and then must be an integral multiple of $2 \pi, A(v)=2 \pi k$; this is precisely the result when petal circles $c_{v_{1}}, c_{v_{2}}, \cdots, c_{v_{n}}$ wrap $\frac{A(v)}{2 \pi}=k$ times about $c_{v}$.

A circle packing for $K$ is computed by finding a label $R$, termed a packing label, with the property that $\theta_{R, \Phi}(v)=A(v)$ for every interior vertex $v$. Typically, the values $R(w)$ for boundary vertices $w$ are prescribed in advance. With label in hand, one can position the circles in the pattern of $K$ to get $P$. 
This positioning stage is a layout process analogous to analytic continuation for analytic functions. Only after the layout does one finally realize circle centers. Our work will be carried out in hyperbolic geometry, where we use the fact that boundary radii may be infinite when associated with horocycles. The various existence, uniqueness, and monotonicity results needed for our applications would hold in euclidean geometry as well.

The Perron method for computing a packing label proceeds via superpacking labels, that is, labels $R$ for which the inequality $\theta_{R, \Phi}(v) \leq A(v)$ holds for all interior $v$ and which has values no less than the designated values at boundary vertices. It is easy to show that the family of superpacking labels is nonempty and that the packing label is the family's infimum. This infimum may be approximated to any desired accuracy by an iterative adjustment process - this is basically how CirclePack computations are carried out. The following condition $(\star)$ is required to ensure non-degeneracy: If $\left\{e_{1}, e_{2}, \cdots, e_{k}\right\}$ is a simple closed edge path in $K$ which separates some edge-connected non-empty set $E$ of vertices from $\partial K$, then the following inequality must hold

$$
\sum_{1}^{k}\left(\pi-\Phi\left(e_{j}\right)\right) \geq 2 \pi+\sum_{v \in E}(A(v)-2 \pi) .
$$

Our work here requires the following extensions to the given data:

- Zero Labels: We will introduce situations in which labels for certain interior vertices go to zero, corresponding with circles that in the final configuration have degenerated to points, namely to their centers. Zero radii actually fit quite naturally into the trigonometric computations, but we will only encounter them for isolated vertices.

- Deep Overlaps: When introducing circle packing, Thurston included specified overlaps $\Phi(e)$, as we do. In general, however, the restriction $\Phi(e) \in[0, \pi / 2]$ is required for existence. We will allow deep overlaps, that is overlaps in $(\pi / 2, \pi]$. Note that overlaps may already be specified as part of the original packing problem under consideration, but these will remain in the range $[0, \pi / 2]$. It is only in the modifications within black holes that deep overlaps may be needed, and these will carry clear restrictions.

- Branching: Traditional branching, described earlier in the paper, is associated with target angle sums $A(v)=2 \pi k$ for $k \geq 2$. These are subject to the condition $(\star)$ noted above, which concerns interactions of combinatorics and angle sum prescriptions. It traces to the simple observation that it takes at least 5 petal circles to go twice around a circle. The tight conditions emerged first in work on branched tangency packings in [5] and [3]. These were modified to incorporate overlaps in 44; Condition $(\star)$ parallels the conditions there while 
allowing equality, which is associated with zero labels in black holes, as we see shortly.

The monotonicities behind the Perron arguments depend on our ability to realize any face $\langle u, v, w\rangle$ with a triple of circles $\left\{c_{v}, c_{u}, c_{w}\right\}$ having prescribed radii and overlaps. To include deep overlaps and zero labels, it is relatively easy to see that some side conditions on $\Phi$ are required. What we need is given in the following lemma, a minor extension of the hyperbolic results in [1.

Lemma 1. Given three hyperbolic radii, $r_{1}, r_{2}, r_{3}$, at least two of which are non-zero, and given three edge overlaps $\phi_{12}, \phi_{23}, \phi_{31} \in[0, \pi]$ satisfying $\phi_{12}+\phi_{23}+\phi_{31} \leq \pi$, there exists a triple $\left\langle c_{1}, c_{2}, c_{3}\right\rangle$ of circles in $\mathbb{D}$ which realize the given radii and overlaps.

The angles $\alpha, \beta, \gamma$ of the triangle $T$ formed by their centers are continuous functions of the radii and overlaps and are unique up to orientation and conformal automorphisms of $\mathbb{D}$. Moreover $\alpha$ is strictly decreasing in $r_{1}$, while area $(T)$ is strictly increasing in $r_{1}$. Likewise, $\beta$ (resp. $\gamma$ ) is strictly increasing in $r_{1}$ (assuming $r_{2}$ (resp. $r_{3}$ ) is finite).

In our generalized branching, zero labels and deep overlaps are temporary devices only within black holes; we modify the combinatorics and set overlap parameters in there to control apparent branch locations. The results, however, are then used to layout a circle packing $P$ for the original complex $K ; P$ itself does not involve any zero labels or deep overlaps, and aside from ambiguity about one circle in the shifted branching case, $P$ is a normal circle packing configuration.

We conclude these preparations by noting the two conditions which are necessary to guarantee existence and uniqueness of the packings. Namely, condition $(\star)$ and this condition $(\star \star)$

$(\star \star) \quad \Phi\left(e_{1}\right)+\Phi\left(e_{2}\right)+\Phi\left(e_{3}\right) \leq \pi$ if edges $\left\{e_{1}, e_{2}, e_{3}\right\}$ form a face of $K$.

With this, we may now describe our two discrete generalized branching mechanisms.

5.2. Singular Branching. Singular branching is used to simulate a branch point lying in an interstice of $P_{K}$. The interstice is defined by a face $\left\langle v_{1}, v_{2}, v_{3}\right\rangle$, corresponding to red, green, and blue circles, respectively, in our illustrations. The black hole is the union of the target interstice and the three interstices sharing its edges. The combinatorics imposed and the event horizon are illustrated in Figure 7. The complex $K$, modified inside the black hole, will be denoted $\widetilde{K}$ and serves as our complex for subsequent computations. The circles of Figure 7 are a device for display only and are 
not part of the final circle configuration. Indeed, before computing the circles of the branched packing we need to prescribe target angle sums, $A$, and edge overlaps, $\Phi$.

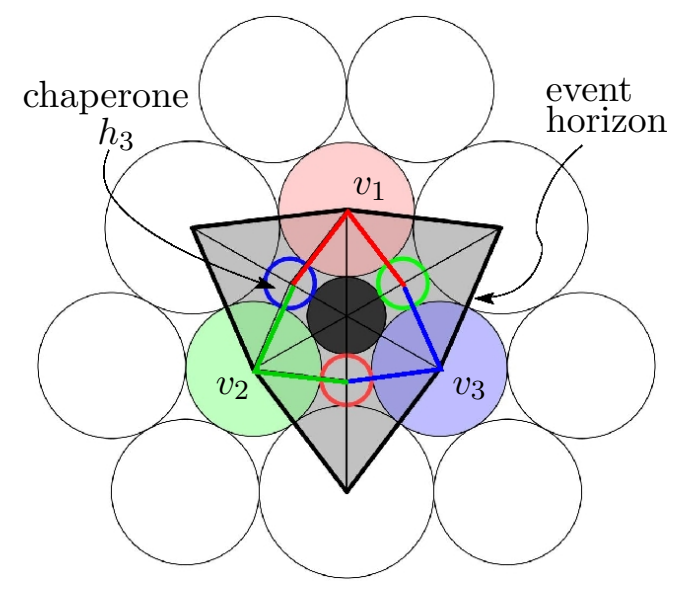

Figure 7. Combinatorics for a singular black hole.

Interior to the event horizon we have introduced 4 additional vertices. Three of these, $h_{1}, h_{2}, h_{3}$, are termed chaperones since they help guide the circles for $v_{1}, v_{2}$, and $v_{3}$; we label $h_{3}$ in Figure 7. A fourth vertex $g$, in the center, is called the fall guy. Specify target angle sums $A(v) \equiv 2 \pi$ for all interior vertices $v \in \widetilde{K}$ with the exception of $g$, setting $A(g)=4 \pi$.

Singular branching is controlled via overlap parameters associated with a partition of $\pi, \gamma_{1}+\gamma_{2}+\gamma_{3}=\pi$. For $i=1,2,3$, the value $\gamma_{i}>0$ represents the overlap angle prescribed in $\Phi$ for the edges from $v_{i}$ to the chaparone circles on either side. These three pairs of edges are color coded in Figure 7 . We set $\Phi(e)=0$ for all other edges of $\widetilde{K}$.

Before describing how these parameters are chosen, observe that we are assured of a circle packing $\widetilde{P}$ for $\widetilde{K}$ with label $\widetilde{R}$, interior angle sums $A$, and overlaps $\Phi$. In particular, if $\Gamma$ denotes the chain of 6 colored faces surrounding the fall guy, $g$, then condition $(\star)$ holds whenever the angle sum prescription $A(g)$ satisfies $A(g) \leq 4 \pi$, with equality when $A(g)=4 \pi$. Traditional Perron and layout arguments imply the existence and uniqueness of the circle packing $\widetilde{P}$ in which the circle for $g$ has radius zero. An example of the result is illustrated in Figure 8. For this we set roughly $\gamma_{1}=0.22 \pi, \gamma_{2}=0.40 \pi$, and $\gamma_{3}=0.37 \pi$.

This image takes some time to understand. The circle for $g$ has degenerated to a point, the branch value, which is at the common intersection point of the circles for $v_{1}, v_{2}, v_{3}$ and also for chaperones $h_{1}, h_{2}, h_{3}$; it is labeled $w$ in the detail zoom. The branching is confirmed in the larger image by observing how the event horizon wraps twice about the branch value. If we 


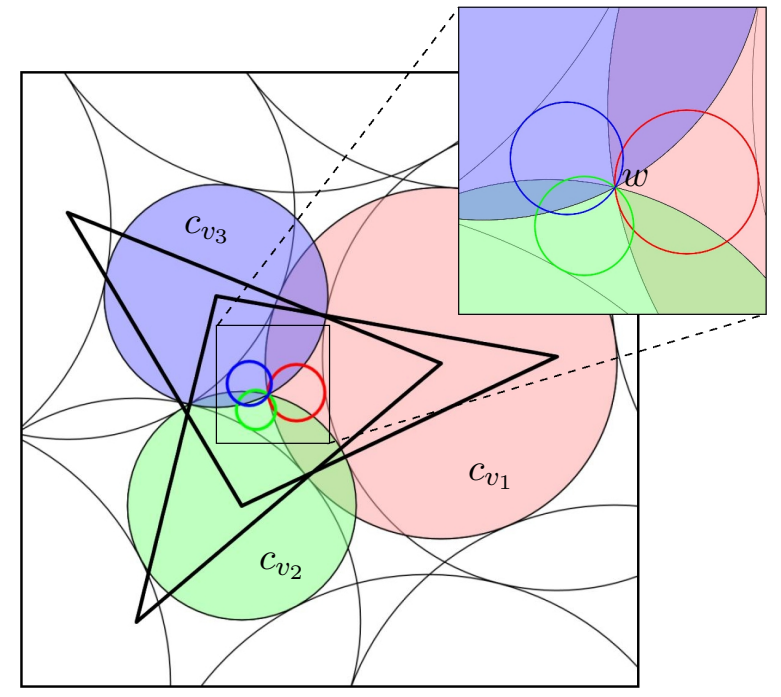

FiguRE 8. Image circle packing in the neighborhood of singular branching, and detail zoom.

disregard the chaperones and the fall guy, the remaining circles of $\widetilde{P}$ realize a tangency circle packing for the original complex $K$. That is, the black hole structure was needed only to guide the layout of the original circles.

This portion of the layout can best be understood as living on a two sheeted surface $S$ branched above $w$. Note, for instance, that the overlap of the red and blue circles is only in their projections to the plane: in actuality, the red part of the intersection is on one sheet of $S$ and the blue is on the other. This shows in the orientation of the red, green, blue, which in projection is the reverse of their orientation in $P_{K}$.

Finally, what about choosing parameters $\gamma_{1}, \gamma_{2}, \gamma_{3}$ to get the desired branch point? Figure 9 illustrates our scheme. We have isolated the interstice formed by circles for $v_{1}, v_{2}, v_{3}$ in $P_{K}$. The dashed circle is the common orthogonal circle through the intersection points and defines a disc $D$ which will be treated as a model of the hyperbolic plane. Point $p$ indicates a location where one might wish to have branching occur. Hyperbolic geodesics connecting $p$ to the three intersection points on $\partial D$ determine angles $\alpha_{1}, \alpha_{2}, \alpha_{3}$, indexed to correspond with the vertices $v_{1}, v_{2}, v_{3}$. We then define $\gamma_{j}=\pi-\alpha_{j}, j=1,2,3$. One has complete freedom to choose $\gamma_{1}$ and $\gamma_{2}$ in this scheme, subject to conditions $\gamma_{1}, \gamma_{2}>0$ and $\gamma_{1}+\gamma_{2}<\pi$. We will be seeing examples for $p$ and the other three red branch points later, in Figure 14.

5.3. Shifted Branching. Shifted branching simulates a branch point lying within an interior circle of $P_{K}$. Of course, when that point is the center, then traditional branching would be the easy choice. This will be incorporated 


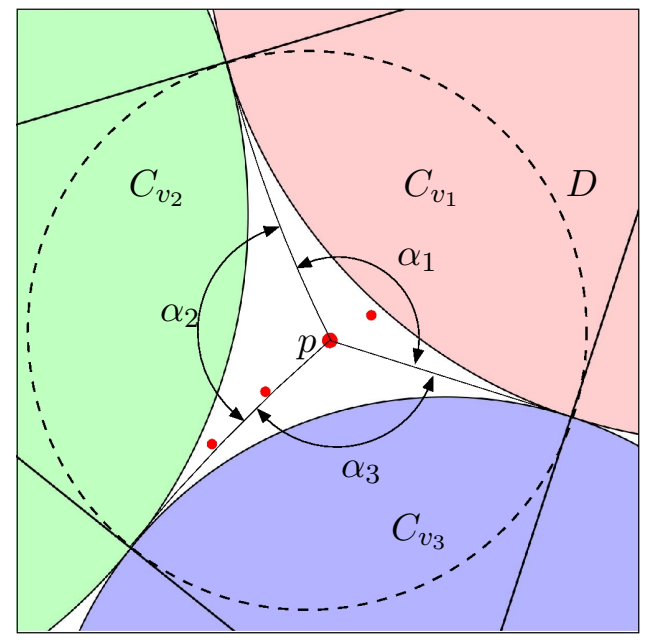

FiguRE 9. The parameter scheme for singular branching.

naturally in our parameterized version, however, so we need not separate out this case.

Suppose $v$ is the interior vertex whose circle is to contain the shifted branch point. The black hole combinatorics shown in Figure 10 are imposed on the flower for $v$. (Note that once again, the circles here are used for display but are not part of our target packing.)

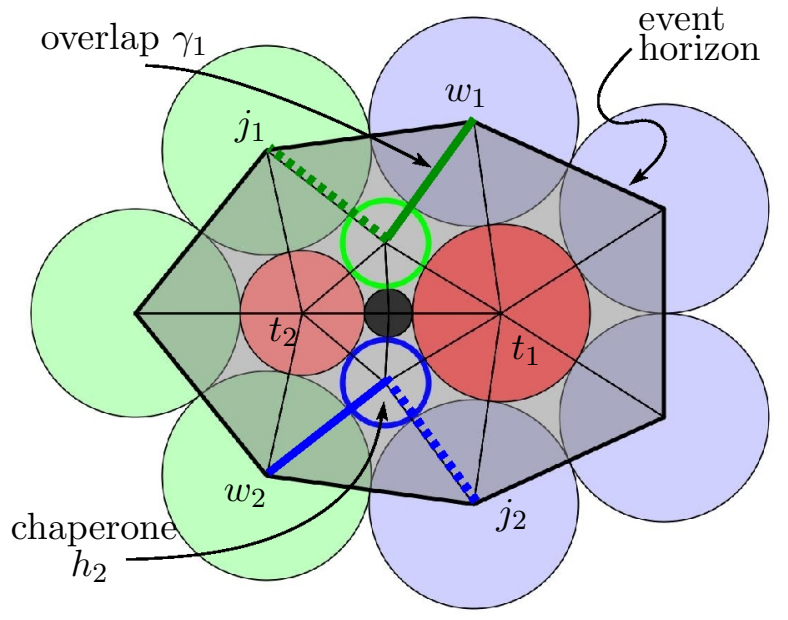

Figure 10. Combinatorics for a shifted black hole.

The event horizon is the chain of edges through the original petals of the flower for $v$ (seven petals, in this case, green and blue). Interior to this horizon, we split $v$, replacing it with the twin vertices, denoted $t_{1}$ and $t_{2}$ and corresponding to the circles in two shades of red. We introduce two chaperone vertices $h_{1}, h_{2}$, respectively green and blue, and a fall guy vertex 
$g$, black; we label only chaperone $h_{2}$ in the figure. With these combinatorics inside the event horizon, we again have a new complex $\widetilde{K}$, for which we need target angle sums $A$ and edge overlaps $\Phi$.

Each chaperone neighbors two original petals, denoted $w_{i}$ and $j_{i}$. The petal $j_{i}$ is known as the jump circle because its chaperone $h_{j}$ and an associated parameter $\gamma_{i}$ facilitate its detachment from one twin and its attachment to the other. The parameters here are $\gamma_{1}$ and $\gamma_{2}$, chosen independently within $[0, \pi]$, and used to define overlaps with the chaperones. In particular, for $i=1,2$, prescribe $\Phi\left(\left\langle h_{i}, w_{i}\right\rangle\right)=\gamma_{i}$ and $\Phi\left(\left\langle h_{i}, j_{i}\right\rangle\right)=\pi-\gamma_{i}$; the edges are shown as solid and dashed lines, respectively, in Figure 10. The other overlaps in $\Phi$ are zero, so Condition $(\star \star)$ holds. Target angle sums are defined as before, namely, $A=2 \pi$ at interior vertices of $\widetilde{K}$, save for the fall guy, with $A(g)=4 \pi$.

Putting aside the choice of jump circles and parameters for now, we are assured of a circle packing $\widetilde{P}$ for $\widetilde{K}$ with label $\widetilde{R}$, interior angle sums $A$, and overlaps $\Phi$. If $\Gamma$ is the chain of edges through the four neighbors of $g$, edges for which tangency is specified, then equality holds in condition $(\star)$, so in $\widetilde{R}$ the radius of the fall guy is necessarily zero.

Figure 11 illustrates the circle packing for $\widetilde{K}$ before we prescribe the branching, in other words, with the target angle sum at $g$ kept at $2 \pi$. We abuse notation by referring to circles by their vertex indices. The original petal circles, starting with $j_{1}$ and ending at $w_{2}$, are shown in green: these are tangent to twin $t_{2}$. Likewise, those starting at $j_{2}$ and ending at $w_{1}$ are shown in blue: these are tangent to twin $t_{1}$.

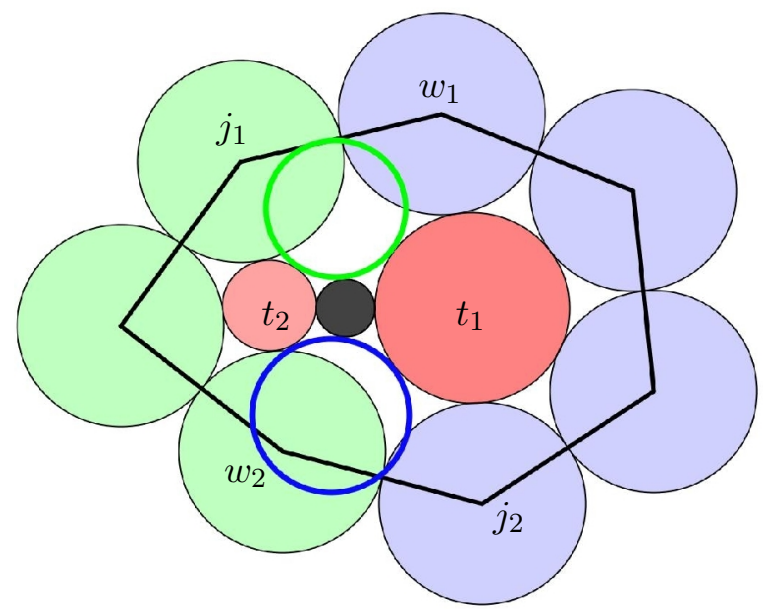

FiguRE 11. The jump circles and parameters are set for a shifted black hole before the branching is imposed. 
We consider the action at chaperone $h_{1}$. First, recall two facts: (1) When a triple of circles has edge overlaps summing to $\pi$, then the three share a common intersection point; and (2) when circles overlap by $\pi$ then one is interior to the other. Here is how the machinery works at $h_{1}$. The circle for $w_{1}$ is tangent to twin $t_{1}, j_{1}$ is tangent to twin $t_{2}$, while $h_{1}$ is tangent to both twins. When $\gamma_{1}=0$, the overlap of $\pi$ between $h_{1}$ and $j_{1}$ forces the jump circle $j_{1}$ to be tangent to $t_{1}$. As $\gamma_{1}$ increases, however, the jump circle separates from $t_{1}$ until, when $\gamma_{1}$ reaches $\pi, w_{1}$ has been pulled in to be tangent to $t_{2}$. In other words, $\gamma_{1}$ acts like a dial: when positive, it detaches the jump circle from $t_{1}$, and as it increases, it moves the jump further around $t_{2}$. The mechanism is similar for chaparone $h_{2}$, as $\gamma_{2}$ serves to detach the jump circle $j_{2}$ from $t_{2}$ and move it further around $t_{1}$.

Typical parameters $\gamma_{1}=0.7 \pi$ and $\gamma_{2}=0.4 \pi$ were specified for Figure 12 . Maintaining these while adding branching at $g$, i.e., setting $A(g)=4 \pi$, gives the configuration of Figure 12. As usual with branching, the image is rather difficult to interpret, so we point out the key features: The twin circles and chaperones are all tangent to $g$, and the radius for $g$ is zero, so these four circles meet at a single point. The twin circles (red) are nested, as are the chaperones (green and blue). The branch value is the white dot in the detail zoom, at the center of the small twin circle and labeled $w$; we explain this shortly. To confirm the topological behavior of generalized branching, note that the circles for the original petals of $v$ wrap twice around $w$ - just follow the image of the event horizon in the larger image as it goes through the petal centers and tangency points. The petals are green and blue in the larger image, corresponding, as in Figure 11, to which twin they are tangent to. The jump circles $j_{1}, j_{2}$ are also labeled.

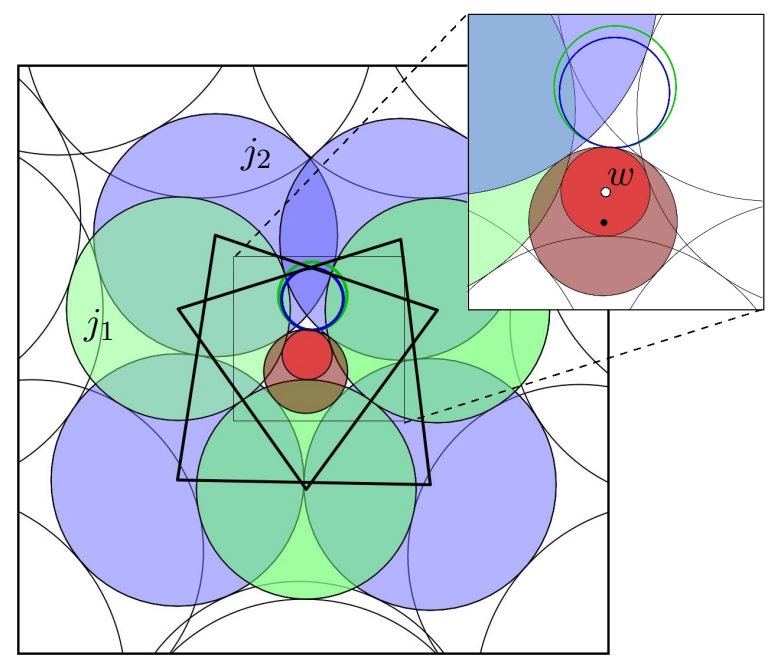

FIGURE 12. Image circle packing in the neighborhood of shifted branching, with detail zoom. 
As with the singular branch image, the configuration of Figure 12 makes sense if one treats it as the projection of circles lying on a two-sheeted surface $S$ branched over $w$. To see this, consider the twins in the detail zoom: $t_{1}$ is the larger twin, with center at the black dot and radius $r_{1}$. The smaller twin has center at $w$ and radius $r_{2}<r_{1}$. Now imagine attaching a string of length $r_{1}$ at the black dot and using it to draw the circle for $t_{1}$ on $S$. As the string sweeps around, it will snag on the white dot at $w$ and, like a yo-yo, trace out the smaller twin on $S$ before finishing $t_{1}$. In other words, the union of the two twin circles together is the projection of all points on $S$ which are distance $r_{1}$ from the center of $t_{1}$ (that is, distance within $S$ ). Exactly this thought experiment was the genesis of shifted branching.

If we disregard the chaperone circles and twins, the remaining circles constitute a traditional tangency circle packing $P$ for $K$, with the caveat that generically the circle for $v$ is ambiguous - neither the circle for $t_{1}$ nor for $t_{2}$ alone can serve as $c_{v}$. We need to live with this ambiguity to achieve the branching behavior we want outside the event horizon. (Having said this, there are (many) settings which lead to identical twin circles, so $P$ then has this common circle as $c_{v}$. All these configurations are identical and are nothing but the circle packing we get when we choose traditional branching at $v$.)

This brings us to the matter of configuring black hole combinatorics and parameters for this shifted branching; that is, choosing the jump circles $j_{1}, j_{2}$ and their associated overlap parameters $\gamma_{1}, \gamma_{2}$. We describe our scheme by referring to Figure 13, which is the flower for $C_{v}$ in $P_{K}$.

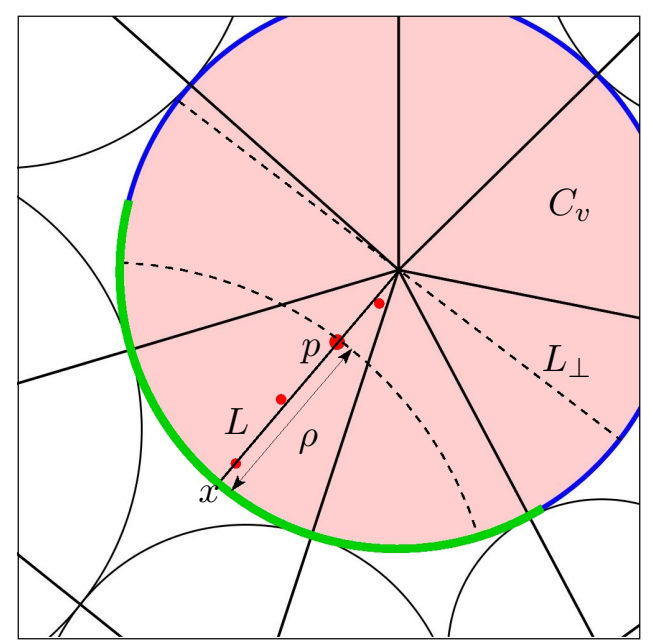

FiguRE 13. Choosing jumps and overlap parameters for shifted branching. 
The ultimate goal is to simulate branching at some point within $C_{v}$, such as the indicated point $p$. In mapping to the branched image packing $P$, the image of the boundary of $C_{v}$ wraps continuously around the boundaries of both twin circles (as we described earlier in referring to the branched surface $S$ ). The jump circles and parameters serve to split the boundary of $C_{v}$ into two arcs, the blue one will be carried to $t_{1}$, the green, to $t_{2}$.

Here we need to observe how the jump and its parameter work together. Recall that in the image packing, $\gamma_{1} \in[0, \pi]$ acts like a dial: The value $\gamma_{1}=0$ forces $j_{1}$ to be tangent to both twins. As $\gamma_{1}$ increases, it pushes $j_{1}$ away from $t_{1}$ and further onto $t_{2}$. When $\gamma_{1}$ reaches $\pi$, it forces the counterclockwise petal $w_{1}$ to become tangent to $t_{2}$. This is a transition point - at this juncture, we could designate $w_{1}$ as the jump circle and reset $\gamma_{1}$ to 0 without altering anything in the image packing. By then increasing the new $\gamma_{1}$ with the new jump circle, we could push yet more boundary onto $t_{2}$. In summary, then, our circle packing map pushes more of $C_{v}$ onto $t_{2}$ by increasing $\gamma_{1}$ and/or moving the designated jump $j_{1}$ clockwise. Likewise, on the other side it pushes more of $C_{v}$ onto $t_{2}$ by decreasing $\gamma_{2}$ and/or moving the designated jump $j_{2}$ counterclockwise.

To illustrate with the point $p$ of Figure 13, the scheme uses the various labeled quantities: The point $x$ where the radial line $L$ from the center of $C_{v}$ through $p$ hits $C_{v}$; the distance $\rho$ from $p$ to $x$; the circular arc (dashed) through $p$ and orthogonal to $C_{v}$; and the diameter $L_{\perp}$ perpendicular to $L$.

To inform our choice of jumps and parameters, we take inspiration from the properties of the branch value $w$ in the eventual image packing - that is, the center of the smaller twin, $t_{2}$. In qualitative terms, the blue arc of $C_{v}$ should map to $t_{2}$, the rest of $C_{v}$ to $t_{1}$. The point $x$ should map to the point of $t_{2}$ antipodal to the tangency point of $t_{1}$ and $t_{2}$. The ratio of $\rho$ to the radius of $C_{v}$ should reflect the ratio of the radii of the two twins. Thus, when $p$ moves close to $C_{v}$, twin $t_{2}$ gets smaller, while as $p$ approaches the center of $C_{v}$, the radius of $t_{1}$ approaches that of $t_{2}$. There is no way to ensure these outcomes precisely - one cannot know, a priori, the outcomes in the image packing, as all the circles get new sizes during computation. We will not burden the reader with the messy details, but we have implemented methods which realize these qualitative behaviors. We illustrate for $p$ and the other three red branch points later, Figure 15.

\section{Fixing An Ahlfors Function}

After successfully constructing a discrete Ahlfors function w for a combinatorial annulus $K$ in $\$ 2.2$, we showed in $\$ 3$ how easily that construction can fail. Making small modifications to $K$ that broke its translational symmetry, we obtained a new combinatorial annulus $K^{\prime}$ which does not support

a discrete Ahlfors function. The problem is non-trivial holonomy, and we 
illustrated in Figure 6 with an attempt at traditional branching using the same midline vertices $v_{1}, v_{2}$ we had used for $\mathbf{w}$.

It seems clear that for $K^{\prime}$ the missing translational symmetry can be blamed for the failure. We now apply the flexibility of generalized branching to repair the damage. Since $K^{\prime}$ still has a midline and reflective symmetry across it, we adopt the following strategy: proceed with traditional branching at vertex $v_{1}$, but use shifted branching near $v_{2}$. Symmetry simplifies our search for the correct branching parameters in the black hole for $v_{2}$ : namely, if we choose vertices $j_{1}$ to be symmetric with $w_{2}$ across the midline, and likewise, $j_{2}$ symmetric with $w_{1}$, and if we specify $\gamma_{2}=\pi-\gamma_{1}$, then the shifted branch value must remain on the midline. After some experimental tinkering, one can in fact annihilate the holonomy and replicate the success we saw for the original complex $K$ - the process works. We do not show the image packing $P$ because it is essentially indistinguishable from Figure 4 . The point is that we are able to make the red cross-cuts coincident.

Admittedly, the fix was (almost) in for this example: we depended on reflective symmetry to reduce the parameter search from a two- to a onedimensional problem. Nonetheless, it demonstrates well the need and potential for generalized branching. We close by discussing the broader issues.

\section{Parameter Space}

This paper is a preliminary report on work in progress. We have focused on generalized branching at a single point $p$ in the interior of $P_{K}$. The location of $p$ is continuously parameterized - e.g, by its $x$ and $y$ coordinates. We have defined discrete generalized branching which seems to handle patches of this parameter space. Thus, when $p$ lies in an interior interstice, singular branching involves two real parameters, $\gamma_{1}, \gamma_{2}$. When $p$ lies in an interior circle, shifted branching involves jump circles and parameters, but in our description of the mechanics it is clear that this, too, is just two real parameters. The continuity of these parameterizations may be phrased in terms of the branched packing labels $R$ restricted to vertices on and outside of the event horizon.

While a proof remains elusive, experiments strongly suggest that this continuity does hold. For example, Figure 14 displays the branched circle packings associated with branching at the four red dots in Figure 9, progressing from lower left to upper right (the third of these is the packing for the distinguished point $p$ from Figure 9). The branch value is roughly at the center in each image. Subject to this and related normalizations, the radii and centers of $P$ appear to be continuous in $\gamma_{1}, \gamma_{2}$.

Figure 15 provides a similar sequence of shifted branched packings for the four red dots of Figure 9 (caution: the chaperones play different roles now). Again we have positioned the branch values roughly at the center in 


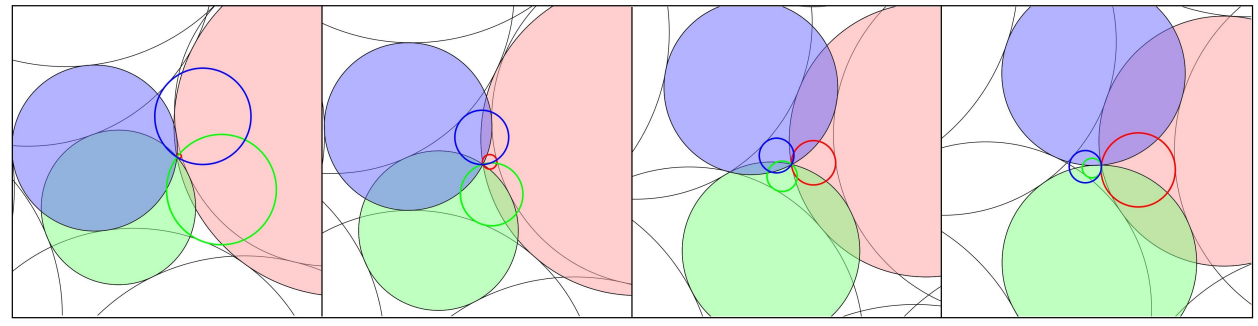

FiguRE 14. Singular branching for the four red branch points of Figure 9 .

each image; the third one corresponds to Figure 12, Here, too, experiments suggest continuity in radii and centers as we manipulate the two shifted branching parameters.

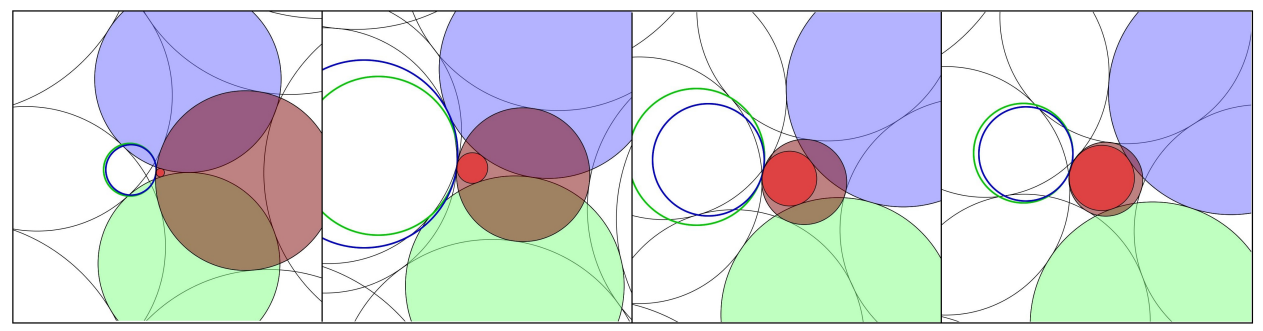

FiguRE 15. Shifted branching for the four red branch points of Figure 13 .

Concatenating the 8 frames in these last two figures highlights another parameterization issue: How are our various patches of parameter space sewn together? If $p$ lies on the mutual boundary of a circle and an interstice, for instance, its generalized branched packing may be treated as a limit of either singular branching from the interstice side or shifted branching from the circle side. We have ad hoc methods for such transitions, though we have yet to formalize the details of parameter alignment. Nevertheless, our images may give a feel for the transition: The interstice formed by $\left\{C_{v_{1}}, C_{v_{2}}, C_{v_{3}}\right\}$ in Figure 9 is contiguous to the circle $C_{v}$ of Figure 13 , that is, $v=v_{1}$. So the 8 frames from Figure 14 and Figure 15 together are part of a movie as the branch point transitions from singular to shifted. Image circles $\left\{c_{v_{1}}, c_{v_{2}}, c_{v_{3}}\right\}$ remain red, green, and blue, respectively, throughout these 8 frames. In the last frame from Figure 14 note that these three appear to be in clockwise order (as we discussed earlier). Compare this to the first frame of Figure 15. the red circle has now split into twins, with the branch value in the smaller twin, so the (small) red, green, and blue are again correctly oriented the branch point has successfully punched though from the interstice to the circle, and in the last frame of Figure 15, it is nearing traditional branching 
at $v$. This is the type of experimental evidence supporting our contention that the two parameter patches can be aligned to maintain continuity.

\section{Global Considerations}

We stated in the introduction that our aim is to bridge the principal gap remaining in discrete function theory, namely the existence and uniqueness of discrete meromorphic functions. Although we have local machinery, we have not confronted the global problem head-on. A few words are in order.

Naturally, one of the first goals would be a more complete theory for discrete rational functions, branched mappings from $\mathbb{P}$ to itself. Here $K$ would be a combinatorial sphere and one would need $2 n$ branch points for a mapping of valence $n+1$. There is a tantalizing approach based on Oded Schramm's metric packing theorem which has motivated some of our work. In [9], Schramm proves remarkable existence and uniqueness results for packings of "blunt disklike" sets - for instance, circles defined using a Riemannian metric on $\mathbb{P}$. Suppose, then, that we are given a classical rational function $\mathbf{F}: \mathbb{P} \rightarrow \mathbb{P}$. We can define a metric $d$ on $\mathbb{P}$ as the pullback under $\mathbf{F}$ of the spherical metric on $\mathbb{P}$. Finding a packing for $K$ by circles in this metric $d$ is tantamount to finding a normal circle packing $P$ on $\mathbb{P}$, and the map from $P_{K}$ to this $P$ would be our discrete rational function. Unfortunately, at the critical values of $\mathbf{F}$ the pullback metric $d$ is not Riemannian; the direct analogue of Schramm's result does not hold, as can be seen, for example, with circles that degenerate. There is still some hope, however, as our constructions demonstrate - the twin circles of a shifted branch point are, after all, the image of a single circle in a pullback metric $d$.

Our hands-on approach still faces many hurdles in practice. On the sphere, for instance, there is no packing algorithm - Perron methods rely on the monotonicity of Lemma 1, which fails in the positive curvature setting. And in other settings, such as Ahlfors and Weierstrass, we have had to depend on symmetry. A generic combinatorial torus $K$ is likely to have no Weierstrass function using traditional branching. Though we believe generalized branching provides the flexibility to overcome the holonomy obstructions, early attempts have faltered due to the curse of (even small) dimension: we don't yet know how to search a two-dimensional space for parameters that will annihilate non-trivial holonomies. We succeeded in the Ahlfors case because partial symmetry reduced us to a one-dimensional search.

We face other global difficulties as well. We list a few. We have restricted attention to simple branching; at least in the case of shifted branching, one can see a chance to allow higher order branching - replacing twins with triplets, etc. In general, one also needs to allow branching at more than one point, but the existence of branched packings then encounters global 
combinatorial issues. The notion of black holes will also need to be extended, since combinatorics may lead to patches of degenerate radii (versus isolated degenerate radii) for branch points in certain combinatorial environments.

In other words, there is considerable work to be done. Nevertheless, we contend that discrete generalized branching addresses - in theory if not in practice - the key obstruction remaining in discrete analytic function theory. This obstruction, of course, is not the only one - so get to work, David.

\section{REFERENCES}

1. James R. Ashe, Generalized branching in circle packing, Ph.D. thesis, Univ. of Tennessee, 2012, (advisor: Ken Stephenson).

2. Alan F. Beardon and Kenneth Stephenson, The Schwarz-Pick lemma for circle packings, Ill. J. Math. 35 (1991), 577-606.

3. Philip L. Bowers, The upper Perron method for labelled complexes with applications to circle packings, Proc. Camb. Phil. Soc. 114 (1993), 321-345.

4. Philip L. Bowers and Kenneth Stephenson, A branched Andreev-Thurston theorem for circle packings of the sphere, Proc. London Math. Soc. (3) 73 (1996), 185-215.

5. Tomasz Dubejko, Branched circle packings, discrete complex polynomials, and the approximation of analytic functions, Ph.D. thesis, University of Tennessee (advisor: Ken Stephenson), 1993.

6. $\_$Branched circle packings and discrete Blaschke products, Trans. Amer. Math. Soc. 347 (1995), no. 10, 4073-4103.

7. David Minda and Burt Rodin, Circle packing and Riemann surfaces, J. d'Analyse Math. 57 (1991), 221-249.

8. Burt Rodin and Dennis Sullivan, The convergence of circle packings to the Riemann mapping, J. Differential Geometry 26 (1987), 349-360.

9. Oded Schramm, Existence and uniqueness of packings with specified combinatorics, Israel J. Math. 73 (1991), 321-341.

10. Kenneth Stephenson, CirclePack software, (1992-), http://www.math.utk.edu/ kens.

11. Introduction to circle packing: the theory of discrete analytic functions, Camb. Univ. Press, New York, 2005, (ISBN 0-521-82356-0, QA640.7.S74).

12. S. Stö̈low, Principes topologiques de la theorie des fonctions analytiques, GauthierVillars, Paris, 1938. 